\title{
Differences between sound scattering by weakly scattering spheres and finite-length cylinders with applications to sound scattering by zooplankton
}

\author{
Timothy K. Stanton \\ Department of Applied Ocean Physics and Engineering, Woods Hole Oceanographic Institution, \\ Woods Hole, Massachusetts 02543-1053 \\ Peter H. Wiebe \\ Department of Biology, Woods Hole, Oceanographic Institution, Woods Hole, Massachusetts 02543-1049 \\ Dezhang Chu \\ Department of Applied Ocean Physics and Engineering, Woods Hole Oceanographic Institution, \\ Woods Hole, Massachusetts 02543-1053
}

(Received 12 August 1996; accepted for publication 26 January 1997)

A modeling study was conducted to determine the conditions under which fluidlike zooplankton of the same volume but different shapes (spherical/cylindrical) have similar or dramatically different scattering properties. Models of sound scattering by weakly scattering spheres and cylinders of finite length used in this analysis were either taken from other papers or derived and herein adapted for direct comparison over a range of conditions. The models were examined in the very low- $(k a$ $\ll 1, k L \ll 1)$, moderately low- $(k a \ll 1, k L \gtrsim 1)$, and high-frequency regions $(k a \gg 1, k L \gg 1)$, where $k$ is the acoustic wave number, $a$ is the radius (spherical or cylindrical) of the body, and $L$ is the length of the cylinders (for an elongated body with $L / a=10$, "moderately low" corresponds to the range $0.1 \leqq k a \leqq 0.5$ ). Straight and bent cylinder models were evaluated for broadside incidence, end-on incidence, and averages over various distributions of angle of orientation. The results show that for very low frequencies and for certain distributions of orientation angles at high frequencies, the averaged scattering by cylinders will be similar, if not identical, to the scattering by spheres of the same volume. Other orientation distributions of the cylinders at high frequencies produce markedly different results. Furthermore, over a wide range of orientation distributions the scattering by spheres is dramatically different from that of the cylinders in the moderately low-frequency region and in the Rayleigh/geometric transition region: (1) the Rayleigh to geometric scattering turning point occurs at different points for the two cases when the bodies are constrained to have the same volume and (2) the functional dependence of the scattering levels upon the volume of the bodies in the moderately low-frequency region is quite often different between the spheres and cylinders because of the fact that the scattering by the cylinders is still directional in this region. The study demonstrates that there are indeed conditions under which different shaped zooplankton of the same volume will yield similar (ensemble average) scattering levels, but generally the shape and orientation distribution of the elongated bodies must be taken into account for accurate predictions. (C) 1998 Acoustical Society of America. [S0001-4966(97)01210-1]

PACS numbers: 43.30.Ft, 43.30.Sf, 43.20.Fn [JHM]

\section{LIST OF SYMBOLS}

A

$a$

$\bar{a}$

$b_{m}^{(s)}$

$\beta_{\text {tilt }}$

$\beta$

$c$

$\Delta$

$f$

$f_{\mathrm{bs}}$

$f^{(\infty)}$

area sphere

$L / a$ radius of sphere or cylinder

average radius

modal series coefficient for homogeneous fluid

tilt angle of infinitessimally thin disk or cross section of body at a particular point on the body axis relative to the incident wave $\left(\beta_{\text {tilt }}=0\right.$ corresponds to broadside incidence to the disk axis at a particular point on the axis)

sound speed

distance that the end of the bent cylinder is bent scattering amplitude

scattering amplitude in backscattering direction form function for an infinitely long cylinder $\gamma_{\kappa}, \gamma_{\rho}$

$h$

$i$

$k$

$\mathbf{k}_{i}$

K

$\kappa$

$L$

$\bar{L}$

$\lambda$

$\mu_{p=2}$

$p_{\text {scat }}$ material property parameters in DWBA formulation

$\rho_{2} / \rho_{1}$

$c_{2} / c_{1}$

$\sqrt{-1}$ unless used as a summation index or subscript to $\vec{k}$

acoustic wave number $(=2 \pi / \lambda)$

wave number vector of incident field

$k \cos \theta$

compressibility

length of body

average length of body

acoustic wavelength

phase advance associated with crossing of caus-

tics $\left[\simeq-(\pi / 2) k_{1} a /\left(k_{1} a+0.4\right)\right]$

scattered pressure 


\begin{tabular}{|c|c|}
\hline$P_{0}$ & incident pressure \\
\hline$r$ & distance between scatterer and receiver \\
\hline$\rho_{c}$ & $\begin{array}{l}\text { radius of curvature of longitudinal axis of uni- } \\
\text { formly bent cylinder }\end{array}$ \\
\hline$\rho$ & mass density \\
\hline $\mathbf{r}_{\text {pos }}$ & position vector of axis of deformed cylinder \\
\hline $\mathbf{r}_{v}$ & position vector of volume \\
\hline $\mathbf{r}_{A}$ & position vector of area \\
\hline $\mathscr{R}_{12}$ & $\begin{array}{l}\text { plane wave/plane interface reflection coefficient } \\
\text { (reflection off medium " } 2 \text { ', due to incident } \\
\text { beam in medium " } 1 \text { "') }\left[=\left(\rho_{2} c_{2} / \rho_{1} c_{1}\right.\right. \\
\left.-1) /\left(\rho_{2} c_{2} / \rho_{1} c_{1}+1\right)\right]\end{array}$ \\
\hline RTS & reduced target strength \\
\hline$s$ & $s_{L} / \bar{L}$ \\
\hline$s_{\theta}, s_{L}$ & $\begin{array}{l}\text { standard deviation of angle of orientation or } \\
\text { length, respectively }\end{array}$ \\
\hline
\end{tabular}

\section{INTRODUCTION}

There has been an evolution of modeling of the scattering of sound by zooplankton in recent years. Up until the mid-1980s, zooplankton had been modeled mathematically almost exclusively as spheres (Greenlaw, 1977, 1979; Johnson, 1977; Holliday and Pieper, 1989; Stanton et al., 1987; and summarized in Holliday and Pieper, 1995). The approaches involving sphere models have seen success as (1) some animals are nearly spherical and (2) the sphere model can be considered to be a "first-order approximation" under some conditions for the very complicated scattering process of animals with more complex shape. However, the shape of some animals deviates significantly from that of a sphere and can possess dramatically different scattering properties under certain conditions. For example, euphausiids and shrimp are quite elongated with length-to-width ratios of order 5 or higher. These animals have recently been modeled as finite length cylinders and it has been shown that the scattering properties are dependent upon shape and distribution of orientation angles (Stanton, 1989; Stanton et al., 1993b; Chu et al., 1993; Demer and Martin, 1995) in addition to material properties, size, and acoustic frequency.

Certain important aspects of our understanding of the scattering of sound by finite cylinders are relatively mature. It is therefore timely to perform a systematic comparison between the scattering by cylinders and spheres under a wide range of conditions. Biomass is an important quantity in zooplankton abundance estimation and acoustic scattering levels are quite often expressed in terms of animal biomass. Thus, for zooplankton acoustics applications and for these comparisons, it is important to formulate the scattering in terms of bodies of the same biomass.

In this paper, various sphere and cylinder models from previous publications are reviewed and others are derived herein. All are written in a form so that direct comparisons can be made analytically under certain limiting conditions. Numerical simulations are performed to provide comparison over a broader range of conditions. Dependence of the scattering upon size, shape, orientation distribution, and acoustic frequency are investigated for the various bodies. Given the

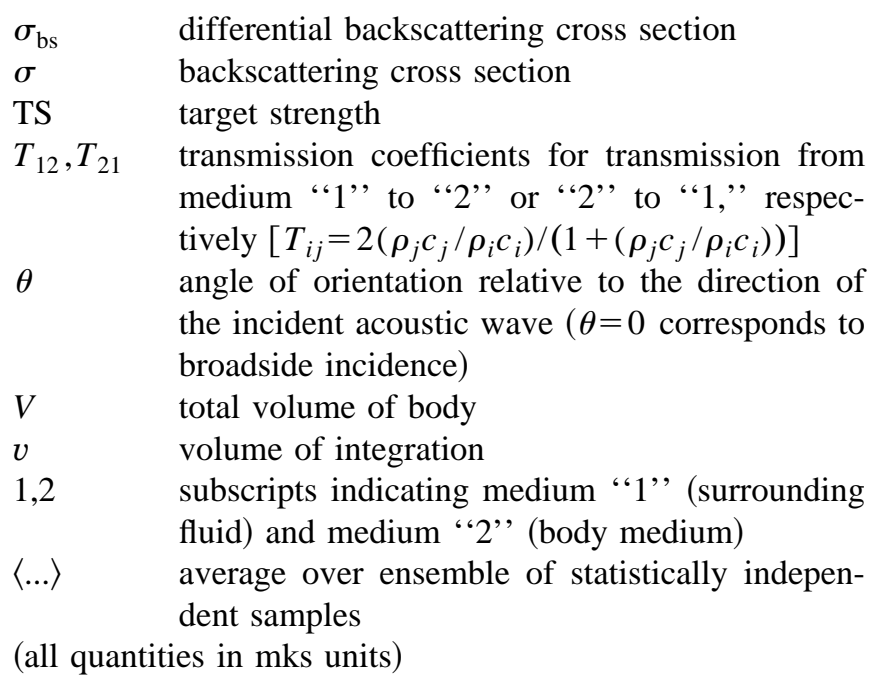

focus on geometrical factors, the dependence upon bulk material properties of the bodies will receive only minor attention. The work is limited to homogeneous weakly scattering bodies that have smooth boundaries. Direct comparisons are made between bodies of the same volume. (Since the animals have mass densities close to that of water, comparisons based on bodies of the same volume are approximately equivalent to comparisons based on bodies of the same biovolume.) The models are presented in terms of both single realizations of size and orientation as well as averages over angles of orientation (in the case of cylinders) and narrow distributions of size. The average over size is performed to relate to either "single-sized" aggregations of zooplankton whose size distribution has a narrow, yet finite width, or a particular size bin of an aggregation with a broader size distribution.

\section{MODELS}

\section{A. Basic quantities}

A fundamental quantity common to all scattering models is the scattering amplitude $f$ which can be defined in terms of the incident and scattered pressures as

$$
p_{\text {scat }}=P_{0} \frac{e^{i k_{1} r}}{r} f \text {, }
$$

where $k_{1}$ is the wave number in the surrounding water (medium " 1 ').

From this definition, the target strength can be defined as

$$
\mathrm{TS}=10 \log \left|f_{\mathrm{bs}}\right|^{2}=10 \log \sigma_{\mathrm{bs}}=10 \log (\sigma / 4 \pi),
$$

where the target strength is also expressed in terms of the two backscattering cross sections that appear in the literature (Urick, 1983; Clay and Medwin, 1977). Here the scattering amplitude is evaluated for the backscatter direction. The units of target strength are $\mathrm{dB}$ relative to $1 \mathrm{~m}^{2}$. The "mean target strength" is based upon the ensemble average of the square of the magnitude of the scattering amplitude:

$$
\langle\mathrm{TS}\rangle=10 \log \left\langle\left|f_{\mathrm{bs}}\right|^{2}\right\rangle \text {. }
$$


Sometimes it is convenient to examine the target strength on a dimensionless scale. The "reduced" target strength RTS normalizes the target strength by the square of some outer dimension of the body:

$$
\begin{aligned}
& \operatorname{RTS}^{(s)}=\mathrm{TS}-10 \log \left(\pi a^{2}\right) \quad(\text { sphere of radius } a), \\
& \operatorname{RTS}^{(e b)}=\mathrm{TS}-10 \log \left(L^{2}\right)
\end{aligned}
$$$$
\text { (elongated body of length } L \text { ). }
$$

\section{B. General models}

There are many approaches to modeling the scattering of sound by objects. The particular approach depends upon the shape and material properties of the body as well as conditions such as frequency range (or more precisely, range of size-to-wavelength ratio). Ideally, one would wish to use an exact model. However, exact solutions to the acoustic wave equation exist only for a small number of shapes, the sphere being one of them. For shapes such as a finite cylinder, an approximate approach is required.

The exact solution for the scattering by a fluid sphere was derived by Anderson (1950) and can be written for the (farfield) backscattering direction as

$$
f_{\mathrm{bs}}=\frac{i}{k_{1}} \sum_{m=0}^{\infty} b_{m}^{(s)}(-1)^{m},
$$

where $b_{m}^{(s)}$ is the modal series coefficient for the fluid sphere and $k_{1}$ is the acoustic wave number in the surrounding fluid medium. This equation is exact for all homogeneous materials that do not support a shear wave (gas or liquid). (The above equation is also written in a general enough form to apply to solid elastic spheres and spherical shells, provided the appropriate modal series coefficients are used.)

For more complex shapes for which there is no exact solution to the wave equation, approximate solutions are required to describe the scattering. The distorted wave Born approximation (DWBA) is a useful formulation as it can predict scattering over the entire range of $k a$ and for arbitrarily shaped bodies at any angle of orientation. It is restricted to weakly scattering materials in that the density and speed of sound of the body must be very close (within about $10 \%$ ) to that of the surrounding medium. Animals like euphausiids fit that requirement as their density and sound speeds are to within several percent of those of the surrounding water. The DWBA is given in general form as (Morse and Ingard, 1968)

$$
f_{\mathrm{bs}}=\frac{k_{1}^{2}}{4 \pi} \iint_{v} \int\left(\gamma_{\kappa}-\gamma_{\rho}\right) e^{i 2\left(\vec{k}_{i}\right)_{2} \cdot \vec{r}_{v}} d v,
$$

where the integration is within the entire body whose volume is described by the position vector $\vec{r}_{v}$. This formula is the complex conjugate of the one presented in Morse and Ingard and is consistent with the phase shift convention $e^{+i k r}$ for an outgoing scattered wave. Also, in this "distorted wave", formulation, the incident wave number vector in the exponent is evaluated inside the body or medium " 2 "' $\left[\left(\vec{k}_{i}\right)_{2}\right]$. This equation is very convenient to perform numerical integrations to check other formulations as well as to be used to derive analytical expressions for scattering (Chu et al., 1993; Stanton et al., 1993b, 1998). The material properties are described by the terms $\gamma_{\kappa}$ and $\gamma_{\rho}$ and are allowed to vary within the body in this formulation. Those parameters can be expressed in terms of the compressibility $\kappa$, mass density $\rho$, density contrast $g$, and sound speed contrast $h$ as

$$
\begin{gathered}
\gamma_{\kappa} \equiv \frac{\kappa_{2}-\kappa_{1}}{\kappa_{1}}=\frac{1-g h^{2}}{g h^{2}}, \\
\gamma_{\rho} \equiv \frac{\rho_{2}-\rho_{1}}{\rho_{2}}=\frac{g-1}{g},
\end{gathered}
$$

where the relation

$$
\kappa=\left(\rho c^{2}\right)^{-1}
$$

and the definitions

$$
h=\frac{c_{2}}{c_{1}}, \quad g=\frac{\rho_{2}}{\rho_{1}},
$$

were used (the " 1 "' subscripts refer to the surrounding water and the " 2 "' subscripts refer to the body). For weakly scattering zooplankton where $g$ and $h$ are each approximately several percent above unity (e.g., 1.04), $\gamma_{\kappa}$ and $\gamma_{\rho}$ are approximately -0.1 and 0.04 , respectively.

For elongated bodies of circular cross section and uniform material properties within any given cross-sectional slice, two of the integrations can be performed analytically, leaving a one-dimensional integral:

$$
\begin{aligned}
f_{\mathrm{bs}}= & \frac{k_{1}}{4} \int_{\mathbf{r}_{\mathrm{pos}}} a\left(\gamma_{\kappa}-\gamma_{\rho}\right) \\
& \times e^{2 i\left(\mathbf{k}_{i}\right)_{2} \cdot \mathbf{r}_{\mathrm{pos}} \frac{J_{1}\left(2 k_{2} a \cos \beta_{\mathrm{tilt}}\right)}{\cos \beta_{\mathrm{tilt}}}\left|d \mathbf{r}_{\mathrm{pos}}\right|,}
\end{aligned}
$$

where $J_{1}$ is the Bessel function of the first kind of order one and the integral is along the axis of the body whose position is described by $\mathbf{r}_{\text {pos }}$ (Stanton et al., 1998). This formulation describes the scattering by deformed finite length cylinders in which the radius of each circular cross section as well as the material properties are allowed to vary with position along the lengthwise axis. The axis of the body is allowed to bend. This formulation is valid for all $k a$ and all angles of orientation, but restricted to weakly scattering materials.

A formulation that is very convenient to use in the geometric scattering region is the Kirchhoff or geometric optics integral (Born and Wolf, 1991; Gaunaurd, 1985). This surface integral is given by

$$
f_{\mathrm{bs}}=\frac{i k_{1}}{2 \pi} \mathscr{R}_{12} \iint_{A}\left(\hat{k}_{i}\right)_{1} \cdot \hat{n}_{A} e^{i 2\left(\mathbf{k}_{i}\right)_{1} \cdot \mathbf{r}_{A}} d A,
$$

where the integral is over the surface described by $\mathbf{r}_{A} \cdot\left(\mathbf{k}_{i}\right)_{1}$ is the incident wave number vector evaluated in medium 1. The " $\wedge$ ", indicates a unit vector and $\hat{n}_{A}$ is the outward normal unit vector to the surface. The plane wave/plane interface reflection coefficient $\mathscr{R}_{12}$ is used in the Kirchhoff approximation that led to this formula and takes into account the penetrability of the material by the following (Clay and Medwin, 1977; Ogilvy, 1991): 


$$
\mathscr{B}_{12}=\frac{g h-1}{g h+1} .
$$

Holding the reflection coefficient fixed during the integration is an approximation as it will, in general, vary with angle of incidence.

Finally, another formulation describing the scattering of sound by deformed cylinders is given by the following line integral (Stanton, 1989, 1992):

$$
\begin{aligned}
f_{\mathrm{bs}}= & \frac{-i}{2 \sqrt{\pi}} e^{i \pi / 4} \int_{\mathbf{r}_{\mathrm{pos}}} f_{\mathrm{bs}}^{(\infty)} \\
& \times\left(k_{1} a \cos \beta_{\mathrm{tilt}}\right)^{1 / 2} e^{i 2\left(\mathbf{k}_{i}\right)_{1} \cdot \mathbf{r}_{\mathrm{pos}}}\left|d \mathbf{r}_{\mathrm{pos}}\right|
\end{aligned}
$$

where the form function $f^{(\infty)}$ for an infinitely long cylinder is used in the integrand. The term $\vec{r}_{\text {pos }}$ is the position vector for the axis. This approximate formulation is valid for all $k a$ and for any material property profile (e.g., fluid, solid elastic, fluid-filled shell, etc.) that is symmetrical about the axis in any given cross-sectional slice. The formulation is only valid for angles near broadside incidence (within about $15^{\circ}$ of broadside for straight cylinders and a wider range for bent cylinders) and for high ratios of length to width (length/width $\gtrsim 5$ ). For a study on the range of accuracies of this model, see Partridge and Smith (1995). Hence two deformed cylinder formulations are provided above. One based on the DWBA weak scattering theory [Eq. (12)] that is valid for all angles of orientation, but is only useful for weakly scattering materials. The other is based upon infinite cylinder form functions [Eq. (15)] and is applicable to a wide range of material properties, but is limited in its usefulness with respect to angle of orientation.

\section{Arbitrarily shaped bodies- $k d \ll 1$}

For weakly scattering bodies of any shape and with all dimensions of the body much smaller than the acoustic wavelength (or more precisely, $k d \ll 1$, where $d$ is the greatest outer dimension of the body such as length), the scattering can quite readily be calculated with the DWBA approach:

$$
f_{\mathrm{bs}}=\frac{k_{1}^{2}}{4 \pi}\left(\gamma_{\kappa}-\gamma_{\rho}\right) V .
$$

In this Rayleigh scattering limit, the scattering amplitude is shown to depend upon the product of the square of the wave number and volume, $V$, of the body. The integral in the general DWBA integral [Eq. (7)] was performed quite readily as the exponent in the integrand was negligibly small and the integral reduced to integrating a constant value (assuming that the material properties were constant inside the body) over the volume. The scattering does not depend upon angle of orientation which is what one would expect in this long wavelength limit. An average of the square of the magnitude of the scattering amplitude over angle of orientation and a distribution of sizes gives, quite trivially,

$$
\left\langle\left|f_{\mathrm{bs}}\right|^{2}\right\rangle=\frac{k_{1}^{4}}{16 \pi^{2}}\left(\gamma_{\kappa}-\gamma_{\rho}\right)^{2}\left\langle V^{2}\right\rangle .
$$

These formulas are convenient for making calculations of the scattering by complex weakly scattering bodies in the long wavelength limit, especially when there is no exact solution for the body of interest. Because of the long wavelength restriction, the usefulness is limited. For objects resembling spheres, the equations are valid for $k a \leqq 0.5$, where $a$ is the equivalent spherical radius. While the scattering levels for spheres are small in this region, they might be detectable. However, for objects that are very elongated such as euphausiids, the usefulness of the equations is more limited than for spheres because of the condition $k L \leqq 1.0$. For elongated bodies with ratios of $L / a$ of the order 5 or greater, this $(k L)$ condition results in the equations being valid only for $k a \leqq 0.2$, where $a$ is the cylindrical radius. In the $k a<0.2$ region, the scattered levels might not be detectable by an echosounder (especially when individuals rather than dense aggregations are involved), hence a more complex approach with fewer approximations needs to be used in calculating the scattering by elongated bodies in the detectable region.

\section{Sphere-single realizations}

For spheres in the $k a \ll 1$ or Rayleigh scattering region, the exact modal series solution can easily be used to predict scattering levels by taking the low $k a$ limit in the modal series terms. In the low $k a$ limit, the first two modes of vibration ( $m=0$ monopole term and $m=1$ dipolelike term) are of the same order of $k a$ and dominate the remaining terms of the series. Keeping only those terms gives the following commonly used expression:

$$
f_{\mathrm{bs}}=a\left(k_{1} a\right)^{2} \alpha_{\pi s}, \quad k_{1} a \ll 1,
$$

where

$$
\alpha_{\pi s} \equiv \frac{1-g h^{2}}{3 g h^{2}}+\frac{1-g}{1+2 g} .
$$

This equation shows that the scattering is a function of a product of the square of the wave number and the cube of the radius (Anderson, 1950). This limiting expression can be compared directly with the DWBA result given in Eq. (16) by writing $a$ in terms of the volume of the body and substituting equivalent expressions for $\gamma_{\kappa}$ and $\gamma_{\rho}$ given in Eqs. (8) and (9) into the DWBA result. The comparison shows that the two approaches produce nearly identical results with the only difference being in the material property term: the denominator $1+2 g$ in the modal-series-based solution is replaced by the term $3 g$ in the DWBA expression (this comparison is made with a factor of $\frac{1}{3}$ moved from outside to inside the parentheses in the DWBA expression for direct comparison). For weakly scattering bodies, $g$ is to within several percent of unity making $1+2 g \approx 3 g \approx 3$.

In the region in which $k a$ is of the order unity or greater, the modal series solution requires more terms to converge and it becomes cumbersome to deal with analytically. Certainly, the solution can be programmed into a computer for numerical results. However, making use of the modal series for analytical means is tedious. One approach to circumvent this problem involves applying the (approximate) Kirchhoff integral in which the scattered field is estimated by summing contributions from the front and back interface of the body in 
the $k a \gg 1$ region. Using the Kirchhoff integral given above, the backscattering by a weakly scattering sphere is approximately

$$
f_{\mathrm{bs}}=\frac{1}{2} a \mathscr{B}_{12} e^{-i 2 k_{1} a}\left(1+T_{12} T_{21} e^{i 4 k_{2} a}\right),
$$

where the transmission coefficients due to the passing of the wave through the front interface (first into the body, then back out of the body) are given as

$$
T_{12}=\frac{2 g h}{1+g h} \simeq 1, \quad T_{21}=\frac{2}{1+g h} \simeq 1 .
$$

The reflection coefficient from the back interface is simply equal to the negative of the one from the front interface and a simple substitution for it in terms of $\mathscr{B}_{12}$ was made.

Here the integral was performed by dividing the integral into two parts, one over the front interface where the Kirchhoff expression was used directly and the other over the back interface where the expression was multiplied by the product $T_{12} T_{21}$ to account for the fact that the wave experiences a slight loss of signal when traveling through an interface. The normal to the surface in each integral is aimed in the general direction of the sound source (i.e., it is the outward normal for the front interface and inward normal for the back interface). The resultant equation [Eq. (20)] shows contributions from both interfaces where the first term (" 1 ") in the parentheses corresponds to the contribution from the front interface and the second term is due to the back interface. The phase shift difference between the echoes from the front and back interfaces is clear in the second term. This phase difference will give rise to interferences between the echoes from the two interfaces. The interference will be constructive or destructive, depending upon the value of $k a$.

\section{E. Sphere-Average echoes}

Since the scattering by spheres does not depend upon the angle of orientation of the sphere, the average over angle of orientation is trivial. Averaging the square of the magnitude of the backscattering amplitude in the $k a \ll 1$ region [Eq. (18)] over a range of sizes is quite simply

$$
\left\langle\left|f_{\mathrm{bs}}\right|^{2}\right\rangle_{a} \simeq k_{1}^{4}\left\langle a^{6}\right\rangle_{a} \alpha_{\pi s}^{2}, \quad k_{1} a \ll 1 .
$$

The bracket $\langle\cdots\rangle_{a}$ denotes the average over a distribution of $a$. For the $k a \gg 1$ region, the average over a narrow Gaussian distribution of sizes using Eq. (20) is

$$
\begin{aligned}
\left\langle\left|f_{\mathrm{bs}}\right|^{2}\right\rangle_{a} & \simeq \frac{1}{2} \bar{a}^{2} \mathscr{B}_{12}^{2}\left[1+e^{-8\left(k_{2} \bar{a} s\right)^{2}} \cos \left(4 k_{2} \bar{a}\right)\right], \quad k_{1} a \gg 1 \\
& \simeq \frac{1}{2} \bar{a}^{2} \mathscr{B}_{12}^{2},
\end{aligned}
$$

where $T_{12} \simeq T_{21} \simeq 1$ was used.

This average shows that the oscillatory effect due to the interference between the two interfaces becomes exponentially small for high $k a$. In the high $k a$ limit, the average backscattering energy is simply equal to the sum of the energy from each interface (the square of the magnitude of the backscattering amplitude from each interface is equal to $\frac{1}{4} \bar{a}^{2} \mathscr{B}_{12}^{2}$ ).

\section{F. Cylinders-Single realizations}

For the backscattering by straight cylinders in the $k a$ $\ll 1$ region, the deformed cylinder formulation has been used to produce the following equation (Stanton, 1988, 1989):

$f_{\mathrm{bs}} \simeq \frac{1}{2}\left(K_{1} a\right)^{2} L \alpha_{\pi c} D_{\mathrm{SC}}(\theta), \quad$ straight cylinder, $k_{1} a \ll 1$,

where the directivity function is given as

$$
D_{S C}(\theta)=\frac{\sin \left(k_{1} L \sin \theta\right)}{k_{1} L \sin \theta}
$$

and the material property term is

$$
\alpha_{\pi c}=\frac{1-g h^{2}}{2 g h^{2}}+\frac{1-g}{1+g} .
$$

Here, the modal-series-based form function of the infinitely long fluid cylinder was used in the calculations. By the nature of this approximation, the result is only valid for near broadside incidence. Calculations for near end-on incidence would depend strongly upon the particular shape of end (flat, pointed, rounded, etc.). It has been convenient to approximate the directivity function in Eq. (26) in terms of a Gaussian function as (Stanton et al., 1993b)

$$
D_{\mathrm{SC}} \simeq e^{-\alpha_{\mathrm{SC}}\left(k_{1} L\right)^{2} \theta^{2}}, \quad \alpha_{\mathrm{SC}} \simeq 0.2 .
$$

The empirical directivity parameter $\alpha_{\mathrm{SC}}$ should not be confused with the material property parameters $\alpha_{\pi s}$ and $\alpha_{\pi c}$. With this expression, averages over orientation can easily be made as shown in a later section (Stanton et al., 1993b).

The same modal-series-based deformed cylinder formulation has also been used to estimate the scattering by bent cylinders. The resultant formula for low $k a$ is derived from Stanton (1989) and Stanton et al. (1993b) as

$$
\begin{aligned}
f_{\mathrm{bs}} \simeq & \frac{1}{2 \sqrt{2}}\left(\rho_{c} \lambda\right)^{1 / 2}\left(k_{1} a\right)^{2} \alpha_{\pi c} D_{B C}(\theta) e^{i \pi / 4}, \\
& \text { bent cylinder, } k_{1} a \ll 1,2 k_{1} \Delta \gg 1,
\end{aligned}
$$

where the directivity function,

$$
D_{\mathrm{BC}}(\theta) \simeq e^{-\alpha_{\mathrm{BC}}\left(2 \theta \rho_{c} / L\right)^{2}}, \quad \alpha_{\mathrm{BC}} \simeq 0.8,
$$

has been added heuristically to include effects due to orientation (Stanton et al., 1993b) ( $\theta=0$ corresponds to the case of "broadside" incidence where the cylinder is bent symmetrically away from the transducer). The empirical directivity parameter $\alpha_{\mathrm{BC}}$ should not be confused with the material property parameters $\alpha_{\pi s}$ and $\alpha_{\pi c}$. Here $\Delta$ is the distance that the end of the cylinder is bent $(\Delta=0$ for a straight cylinder). This directivity function is based upon a reasonable estimate of the angle beyond which the scattering decreases dramatically with angle. However, it does not provide accurate estimates of the scattering for near end-on incidence, which, as discussed in the above straight cylinder case, depends strongly upon the particular shape of the end. The function is convenient for averages over orientation as discussed in a later section.

In the $k a \gg 1$ case, the modal-series-based approach becomes difficult to manipulate algebraically because of the 
fact that it takes many terms for the series to converge. Several approaches can be used in this case- the DWBA, Kirchhoff, or form-function-based deformed cylinder solution. In a recent study, a ray-based (form function) deformed cylinder formulation was used to produce the following equation for the straight cylinder and $k a \gtrsim 0.1$ (Stanton et al., 1993a, 1993b):

$$
\begin{aligned}
f_{\mathrm{bs}}= & \frac{-i}{2 \sqrt{\pi}} e^{i \pi / 4} e^{-i 2 k_{1} a} L \sqrt{k_{1} a} \mathscr{B}_{12} D_{\mathrm{SC}}(\theta) I_{0}, \\
& k_{1} a \gtrsim 0.1,
\end{aligned}
$$

where

$$
I_{0}=1-T_{12} T_{21} e^{i 4 k_{2} a} e^{i \mu_{p=2}\left(k_{1} a\right)}
$$

and

$$
\mu_{p=2}\left(k_{1} a\right)=-(\pi / 2) k_{1} a /\left(k_{1} a+0.4\right) .
$$

The term $\mu_{p=2}\left(k_{1} a\right)$ was added heuristically to remove certain phase effects so that the formula, normally valid only for $k a \gg 1$, could be applied to values of $k a$ down to about 0.1 . In this formulation, a ray-based form function for the infinitely long fluid cylinder as presented in Marston (1992) was incorporated into the deformed cylinder formulation. Equation (31) is broadly similar to that of the sphere for $k a \gg 1$ in that two terms appear, one corresponding to the echo from the front interface of the body and the other due to the back interface. They differ greatly due to the dependence of the scattering by the cylinder upon $k a$ and orientation.

For the uniformly bent cylinder, the same ray-based deformed cylinder formulation as described above is applied to the bent cylinder geometry (Stanton et al., 1993a, 1993b). The result of that analysis is

$$
f_{\mathrm{bs}}=\frac{1}{2}\left(\rho_{c} a\right)^{1 / 2} \mathscr{B}_{12} e^{-i 2 k_{1} a} D_{\mathrm{BC}}(\theta) I_{0},
$$

where the directivity term has the same limitations as in the $k a \ll 1$ case.

\section{G. Cylinders-average echoes}

Averaging the square of the magnitude of the backscattering amplitude over angle of orientation takes advantage of the Gaussian form of the above-mentioned directivity functions. Averaging over both angle of orientation and a narrow Gaussian distribution of size results in the following set of expressions:

$$
\begin{aligned}
\left\langle\left|f_{\mathrm{bs}}\right|^{2}\right\rangle_{L, \theta}= & A_{i j} \pi\left(k_{1} \bar{a}\right)^{3} \bar{a} \bar{L} \alpha_{\pi c}^{2}, \quad k_{1} a \ll 1, k_{1} L \geqslant 1, \\
\left\langle\left|f_{\mathrm{bs}}\right|^{2}\right\rangle_{L, \theta}= & 2 A_{i j} \mathscr{B}_{12}^{2} \bar{a} \bar{L}\left[1-e^{-8\left(k_{2} \bar{a} s\right)^{2}}\right. \\
& \left.\times \cos \left(4 k_{2} \bar{a}+\mu_{p=2}\right)\right], \quad k_{1} a \gtrsim 0.1, \\
\left\langle\left|f_{\mathrm{bs}}\right|^{2}\right\rangle_{L, \theta} \simeq & 2 A_{i j} \mathscr{B}_{12}^{2} \bar{a} \bar{L}, \quad k_{1} a \gg 1,
\end{aligned}
$$

where the term $A_{i j}$ takes on different values for different combinations of shapes and orientation conditions (straight/ bent cylinder, Gaussian/uniformly distributed orientation angle). Because Eq. (36) involves an extension into the $k a$ $<1$ region, there is overlap in the frequency regions in which Eqs. (35) and (36) can be used. Equation (36) is from
Stanton et al. (1993b). Equation (35) was derived here in the same manner as Eq. (36). Since $A_{i j}$ was verified numerically down to about $k a=0.1$ in Stanton et al. (1993b), the same $A_{i j}$ are used in both Eqs. (35) and (36). For the case of bent cylinders with a Gaussian distributed orientation angle, $A_{i j}$ $=T_{B}^{2} C_{B}^{2} \mathbf{S}_{\theta} /\left(16 \sqrt{\alpha_{B}} S_{\theta}\right) . A_{i j}$ for other cases are given in Table I of Stanton (1993b). $C_{B}$ is an empirical parameter and is approximately equal to 1.2 while $T_{B}$ in this case is equal to unity. $\mathbf{S}_{\theta}$ is a complex function of the width of the main lobe of the scatter pattern and orientation distribution parameters (Stanton et al., 1993b). For orientation distributions that are wide enough so that the entire main lobe is "seen" by the receiver over the course of the averaging, then $\mathbf{S}_{\theta} \sim 1$. The approximation $T_{12} \simeq T_{21} \simeq 1$ was used in Eq. (36).

Each formula involving a Gaussian distribution of orientation angles assumes that the bell part of the Gaussian distribution contains the broadside angle. It is this assumption that allows use of the Gaussian form of the directivity function. If the broadside angle is part of the averages, then the resultant levels near broadside will dominate the small nearend-on levels. Hence, errors in the end-on levels are not significant in this case. Various numerical simulations involving the more precise DWBA approach support this assumption (Stanton et al., 1993b).

\section{H. Average scattering by targets of equal volume}

Some of the above formulas for averaged echoes are now reformulated so that they can be compared with each other. As discussed above, an important quantity in zooplankton studies is biomass, which is directly proportional to the volume of the animal. The scattering formulas are therefore reformulated in terms of the volume of the body.

For arbitrarily shaped objects in the low-frequency region, Eq. (17), which describes the average square of the magnitude of the backscattering amplitude (or average backscattering cross section), can be used directly from the above analysis without modification. The formula is valid for weakly scattering bodies where the wavelength is much longer than any dimension of the body (or more precisely, $k d \ll 1)$.

At moderately low frequencies where $k a \ll 1$ but $k L \gtrsim 1$ for cylinders, Eq. (17) does not apply (although it is still valid for spheres). In the moderately low-frequency case for cylinders [Eq. (35)], the relationship for volume $V=\pi a^{2} L$ is used along with $\beta=L / a$ to obtain the following formula:

$$
\begin{aligned}
\left\langle\left|f_{\mathrm{bs}}\right|^{2}\right\rangle= & \left(\frac{A_{i j} \alpha_{\pi c}^{2}}{(\pi \beta)^{2 / 3}}\right) k_{1}^{3} V^{5 / 3}, \\
& \text { all cylinders, } \quad k_{1} a \ll 1, \quad k_{1} L \gtrsim 1,
\end{aligned}
$$

where now the average backscattering levels from cylinders depend upon the product of $k^{3} V^{5 / 3}$. For an object with $L / a$ $=10$, these moderately low frequencies are in the range 0.1 $\leq k a \leq 0.5$. As with Eq. (35), this equation is restricted to the cases in which the main lobe of the scattering pattern faces the receiver during part of the averaging. 
TABLE I. Functional dependencies of averaged backscattering upon wave number and volume for spheres and cylinders. Actual scattering levels also depend upon material properties and (for cylinders) distribution of angle of orientation. The angular distributions for the cylinders in the $k L \gtrsim 1$ region are restricted to the case where the main lobe of the scatter pattern is included in the average. The averages over size are for a narrow distribution of size. Volume dependence of scattering will change for cylinders for certain other distributions of angle of orientation in the $k L \gtrsim 1$ region.

\begin{tabular}{llcc}
\hline \hline & \multicolumn{3}{c}{$\begin{array}{c}\text { Cylinders } \\
\text { straight and bent } \\
\left\langle\left|f_{\mathrm{bs}}\right|^{2}\right\rangle_{L, \theta} \\
(0 \leqslant \theta \leqslant 2 \pi)\end{array}$} \\
\hline$k a \ll 1$ & $k L \ll 1$ & $\begin{array}{c}\text { Sphere } \\
\left\langle\left|f_{\mathrm{bs}}\right|^{2}\right\rangle_{a}\end{array}$ \\
$k a \gg 1$ & $k L \gtrsim 1$ & $k^{4} V^{2}$ & $k^{4} V^{2}$ \\
\hline \hline
\end{tabular}

In the high-frequency region where $k a \gg 1(k L$ is, of course, much greater than unity in this region also), the corresponding average echo formulas are reformulated in terms of the volume of the body to obtain

$$
\begin{aligned}
\left\langle\left|f_{\mathrm{bs}}\right|^{2}\right\rangle_{a} & \simeq \frac{1}{2}\left(\frac{3}{4 \pi}\right)^{2 / 3} \mathscr{R}_{12}^{2} V^{2 / 3}, \quad \text { sphere, } k_{1} a \gg 1, \\
& \simeq 0.19 \mathscr{R}_{12}^{2} V^{2 / 3}
\end{aligned}
$$

and

$$
\begin{aligned}
\left\langle\left|f_{\mathrm{bs}}\right|^{2}\right\rangle_{a, \theta} \simeq & \frac{2}{\pi^{2 / 3}} A_{i j} \beta^{1 / 3} \mathscr{R}_{12}^{2} V^{2 / 3} \\
& \text { all cylinders, } k a \gg l, \\
\simeq & \frac{T_{B}^{2} C_{B}^{2} S_{\theta}}{8 \sqrt{\alpha_{B}} \pi^{2 / 3}} \frac{\beta^{1 / 3}}{s_{\theta}} \mathscr{R}_{12}^{2} V^{2 / 3}, \\
& \text { bent cylinder, Gaussian distributed } \theta, k a \gg 1, \\
\simeq & 0.094 \frac{\beta^{1 / 3}}{s_{\theta}} \mathscr{R}_{12}^{2} V^{2 / 3},
\end{aligned}
$$

where the same orientation restrictions apply to Eqs. (41a)(41c) as for Eqs. (35)-(37). Equations (39) and (40) were based on an average of Eq. (24); Eq. (41a) was based on an average of Eq. (37); Eq. (41b) used an $A_{i j}$ element from Table I of Stanton et al. (1993b); and $T_{B}=1, \alpha_{B}=0.8, C_{B}$ $=1.2$, and $S_{\theta} \sim 1$ were used for Eq. (41c) and were taken from Stanton et al. (1993b).

For the case of euphausiids where $\beta \approx 16$ and $s_{\theta}$ $=0.349 \mathrm{rad}\left(20^{\circ}\right)$, Eq. (41c) can be further reduced to

$$
\begin{aligned}
\left\langle\left|f_{\mathrm{bs}}\right|^{2}\right\rangle_{a, \theta} \simeq & 0.68 \mathscr{R}_{12}^{2} V^{2 / 3}, \\
& \text { euphausiid, } \pm 20^{\circ} \text { motion, } k a \gg 1 .
\end{aligned}
$$

The motion is distributed about an arbitrary mean angle provided that the main lobe of the scatter pattern is "seen" by the transceiver within the range of motion. As can be seen in the above equations in Secs. IC-H, while the formulas for single-realization broadside echoes from the various cylinders and spheres depart from each other (except at very low frequency where shape is not a factor), the averaged echoes are functionally very similar under certain conditions (Table

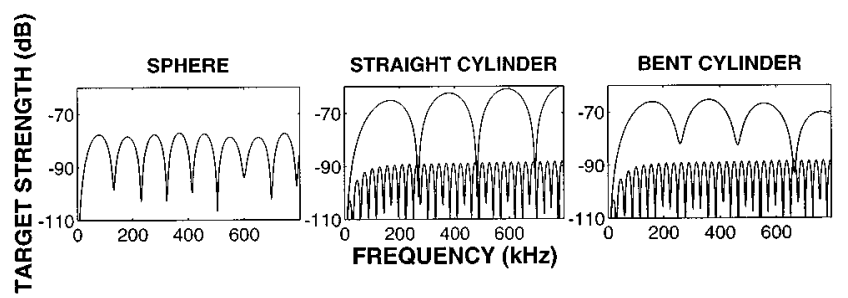

FIG. 1. Theoretical target strength versus frequency for one ping each off of an individual sphere, straight cylinder, and bent cylinder. All bodies have the same volume of $0.30 \mathrm{~cm}^{3}$, which corresponds to a 34-mm-long euphausiid. The upper curves in the cylinder plots are for broadside incidence and the lower plots are for end-on incidence. The acoustic or "reduced" length of the animal is $29 \mathrm{~mm}$, the cylindrical radius is $1.82 \mathrm{~mm}$ for the cylinder model, and the equivalent spherical radius is $4.16 \mathrm{~mm}$ for the sphere model. The length is reduced to account for the fact that the 5-mm telson or "tail-section" of the animal is thin and probably does not scatter much sound. The exact modal-series solution was used for the sphere case [Eq. (6)] and the DWBA method was used for both cylinders [Eq. (12)]. For all plots, $g=1.0357$ and $h=1.0279$ [these values were taken from Foote et al. (1990) and Foote (1990), respectively, as they were measured directly from live euphausiids]. For the bent cylinder $\rho_{c} / L=3.0$ (this value for curvature is chosen as it is a reasonable representation of the degree of bend for a fully extended euphausiid). All objects have a smooth boundary and homogeneous material properties.

I). There are significant deviations in scattering levels in the moderately low-frequency case and high-frequency case for certain ranges of orientation distribution.

\section{NUMERICAL EXAMPLES}

\section{A. Bodies of fixed volume and material properties}

Numerical evaluation of some of the general solutions allows examination of the scattering properties over a wide range of conditions. The exact modal series solution for the fluid sphere and the DWBA integral are used in numerical calculations of backscattering by fluid spheres and straight and uniformly bent cylinders, respectively (Figs. 1-3). All bodies have smooth boundaries and homogeneous material properties. Calculations involving the cylinders were done for fixed angle of orientation (Fig. 1) and distributions of orientation angle (Figs. 2 and 3). The mean angles of $20^{\circ}$ and $45^{\circ}$ and standard deviations of $20^{\circ}$ in Fig. 2 were chosen to represent swimming krill insonified by a downward looking echo sounder (Kils, 1981; Endo, 1993; Miyashita et al., 1996). The mean angle of $90^{\circ}$ was chosen to represent certain elongated animals that would be swimming toward or away from a downward looking sounder such as during diurnal migration. The uniform distribution of angles represents the case in which the sounder is looking sideways and there is no preferred swimming direction in the horizontal plane. Since volume (or biomass) of zooplankton is of particular interest, the volume of each object (spheres and cylinders) is held fixed at $0.30 \mathrm{~cm}^{3}$ as other parameters such as frequency are varied. This volume corresponds to a 34-mmlong euphausiid (a shrimplike animal) whose length-to-width ratio is about 8 . The material properties, density and sound speed contrast, were also chosen to resemble those of a euphausiid. The radius of the sphere is considered the "equivalent spherical radius" of the animal. 


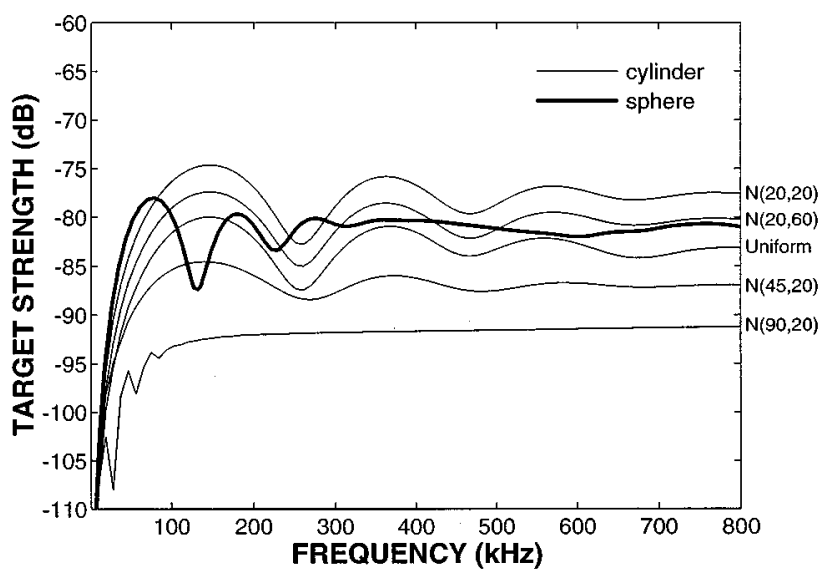

FIG. 2. Average theoretical target strength versus frequency for scattering by statistical ensemble of spheres and bent cylinders. The target strength was averaged on a linear scale as described in Eq. (3) for the models used in Fig. 1. The scattering by all bodies is averaged over a narrow Gaussian distribution of sizes (s.d. of Gaussian is $10 \%$ of mean body length or diameter, averaging is done over the range, mean size \pm 2 s.d.). In addition, the cylinders are also averaged over various normal distributions $[N(\bar{\theta}$, s.d. of $\theta)]$ of angle of orientation and over a uniform $[0,2 \pi]$ distribution in one case. $\theta=0^{\circ}$ corresponds to broadside incidence and $\mathrm{N}\left(90^{\circ}\right.$, $20^{\circ}$ ) is a distribution centered about end-on incidence. The models, body dimensions, and values of $g, h$, and $\rho_{c} / L$ are the same as in Fig. 1. Units of all angles in figure are in degrees.

In general, the overall levels of the scattering by the cylinders depend upon the distribution of orientation angle, especially at the higher frequencies (Fig. 2). Some distributions will produce scattering levels close to that of the sphere, while others will cause it to deviate significantly (of the order $3-10 \mathrm{~dB})$. At the very low frequencies, all models converge to the same levels.

The scattering properties of all objects under investigation are characterized by a Rayleigh scattering region $(\mathrm{ka}$ $\ll 1$, where $a$ is either the spherical or cylindrical radius of the body). Also, each object possesses a geometric scattering

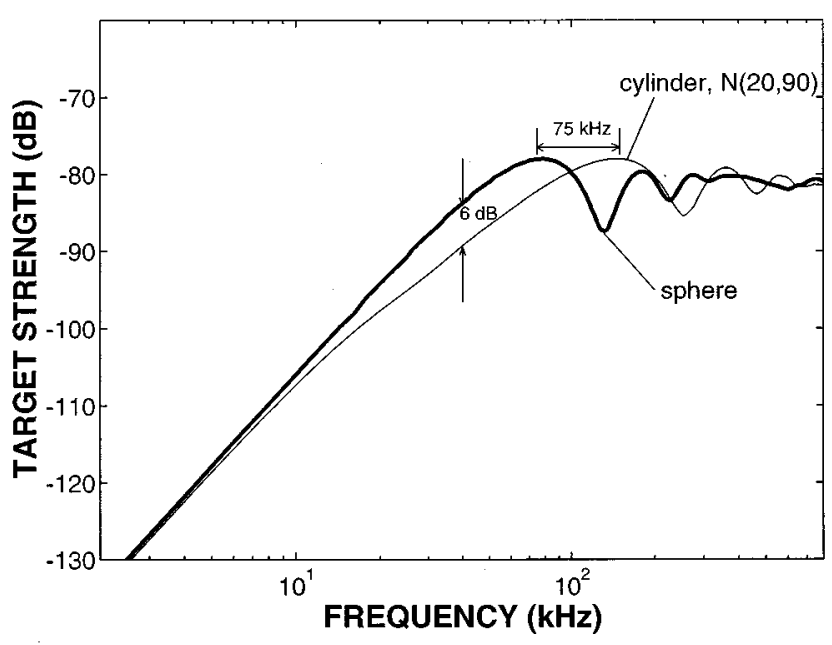

FIG. 3. Comparison between average theoretical target strength of sphere and bent cylinder under conditions where the predictions were close to each other at high frequencies. Averaging performed over narrow Gaussian distribution of sizes for both the sphere and cylinder (as described in Fig. 2) and angle of orientation (cylinder only). The models, body dimensions, and values of $g, h$, and $\rho_{c} / L$ are the same as in Fig. 1. region ( $k a \gg 1)$. Although the transition or "turning" point at which the scattering changes from Rayleigh to geometric occurs at $k a \simeq 1$ for both spheres and cylinders at broadside incidence, the point occurs at different frequencies for those bodies once the volume is held constant (the " $a$ " is different for the sphere and cylinders). For example, for bodies of the same volume of $0.30 \mathrm{~cm}^{3}$, the point occurs at about $75 \mathrm{kHz}$ for the sphere and at about $150 \mathrm{kHz}$ for the cylinders whose orientation is at broadside incidence or includes broadside in the averages (Fig. 3). The turning point for end-on cylinders is at a lower frequency than for the cylinders at broadside incidence.

In addition, the target strength versus frequency pattern for each object possesses an oscillatory pattern in the geometric scattering region. These oscillations are especially pronounced when only single echoes are examined (Fig. 1). An interesting trend occurs in the geometric region of the straight cylinder at broadside incidence where the trend of scattering increases with frequency.

The levels of the backscattering for end-on incidence are markedly lower for these elongated bodies than for the broadside cases (Fig. 1). The level of the scattering in this case is strongly dependent upon the shape of the end of the body [rounded in this case according to the equation $a(z)$ $=a_{0} \sqrt{\left(1-(2 z / L)^{10}\right)}$, where $z$ is the position along the axis ( $z=0$ is the center of the body and $z= \pm L / 2$ at the ends) and $a_{0}=a(0)$ is the (maximum) radius at the middle of the tapered body.] Note also that boundary roughness and material heterogeneities also strongly influence backscattering for end-on incidence (Stanton et al., 1998).

Once the echoes are averaged over a range of orientations and sizes, some of the differences between the scattering by the sphere and cylinders tend to diminish (Figs. 2 and 3 ). For example, for distributions of orientation that include broadside incidence in the bell part of the distribution, the average backscattering by all bodies at high frequencies ( $k a \gg 1$ or frequencies much greater than $150 \mathrm{kHz}$ in this example) tends to be nearly constant with respect to frequency and within about a 10-dB range of values [Fig. 2 and other calculations, such as $\mathrm{N}\left(0^{\circ}, 20^{\circ}\right)$, not shown]. Average scattering levels for straight cylinders have been shown to be quite similar to those of bent cylinders over a wide range of conditions (due to conservation of energy) and are not shown in this paper (Stanton et al., 1993b).

\section{B. Bodies with other volumes and/or material properties}

For bodies with different volumes and/or material properties, predictions (not shown) are broadly similar in form (but with different magnitudes) to those given in Figs. 1-3. For example, for an animal $\frac{1}{10}$ the length of the 34-mm-long one simulated in the figures (and $\frac{1}{10}$ the width, correspondingly), all curves would shift down uniformly by $20 \mathrm{~dB}$ and to the right by one decade of frequency. This uniform shift comes about by the fact that the square of the diameter (sphere) or square of the length (cylinder) can be factored out of each prediction of backscattering cross section. Furthermore, the predictions can be expressed in terms of the dimensionless product $k a$. As a result, predictions are quite often presented in terms of reduced target strength (i.e., nor- 
malized by the square of a dimension) versus $k a$ (Stanton, 1988 , 1989). The RTS plots are valid only when the absorption of sound within the bodies is negligible (absorption does not scale according to wavelength and body size). It is also particularly useful when the ratios of length-to-width and length-to-radius-of-curvature of the lengthwise axis of the elongated bodies remain fixed for a given RTS plot. The last set of conditions is a restriction of self-similarity of the object shape which is reasonable for marine organisms.

For weakly scattering bodies of different material properties, the changes are related to differences between the material properties (or their products) and unity [e.g., Eqs. (7)(9) of this paper; Anderson, 1950; Greenlaw, 1977]. Repeating scattering calculations in this paper for different density and sound speed contrasts ( $g$ and $h$, respectively), the levels increase by about $10 \mathrm{~dB}$ when $(g, h)=(1.1,1.1)$ and decrease by about $10 \mathrm{~dB}$ for $(g, h)=(1.01,1.01)$ when compared with calculations in this paper which used $(g, h)$ $=(1.0357,1.0279)$. The locations of the peaks and dips in the TS versus frequency plots shifted horizontally (somewhat) as the material properties were varied.

\section{DISCUSSION}

The similarities and differences between the various scattering predictions can be explained in terms of basic scattering principles.

In the very-low-frequency case in which all dimensions of the bodies are much smaller than a wavelength, the models show that the scattering levels for the sphere, straight cylinder, and bent cylinder are the same. This is due to the fact that in this very-low-frequency region, the phase of the echo from each part of the body is the same, regardless of position within the body. The scattered levels then depend only upon the volume of the body, regardless of the shape.

In the case of moderately low frequencies (i.e., $k a \ll 1$ and $k L \gtrsim 1$ ), the length of the cylinders plays a role. Because of phase variabilities of the echoes along the length of the elongated bodies, the scattering becomes dependent upon shape and orientation of the cylinders. This dependence occurs in spite of the fact that $k a \ll 1$ where the scattering is in the Rayleigh region with respect to the radius of the body. Here, the phase variabilities are small across any given crosssectional slice of the bodies. However, for the bent cylinder, the phase will vary along the length of the body, regardless of orientation. Furthermore, for orientations of the straight cylinder away from broadside incidence, the phase will vary along the length of that body as well.

The transition or "turning point" from Rayleigh to geometric scattering is different for spheres versus cylinders having the same volume. For broadside incidence or averages over a wide range of angles of orientation, it depends upon the cross-sectional radius of the body. For bodies of constant volume, the radius of the spherical body is about two times bigger than the (cylindrical) radius of the cylinder whose length to (cylindrical) radius is 16 (i.e., for euphausiids). For end-on incidence, the turning point of the cylinders depends upon the length which, in this case, is much greater than the radius of the sphere of the same volume.
In the geometric scattering region, the phase varies within each cross-sectional slice of each body (sphere and cylinders) as well as along the length of the elongated bodies (except for the straight cylinder at broadside incidence). The complex phase variabilities give rise to both an oscillatory pattern in the target strength versus frequency plots as well as a trend in that pattern that depends upon shape. The oscillatory pattern is due to the fact that there is more than one echo coming from the body. A small fraction of the energy of the incident acoustic signal will reflect off an interface facing the acoustic source. However, since this is a weakly scattering body, most of the incident acoustic signal passes into the body relatively unaffected. The internal acoustic signal will then reflect off of an interface that is facing away from the sound source and reflect back toward the source. These two echoes will interfere constructively or destructively according to the value of the separation of the faces with respect to the wavelength of the sound. For a sphere, the two faces are simply the front and back interface of the body, regardless of orientation. For each type of cylinder at broadside incidence, the interferences correspond to echoes from the front and back portions of the body cross section, while for end-on incidence the echoes from these extended bodies come from the front and back ends of the bodies. The period of oscillation of the target strength versus frequency curves is related to the radius of the sphere and cylindrical radius of the cylinders at broadside incidence, while it is related to the length of the cylinders at end-on incidence.

The trend of the oscillations depends upon whether or not the object is curved in one or two dimensions. For spheres and bent cylinders at broadside incidence, the trend is constant with respect to frequency. This is related to the fact that both are curved in two dimensions. However, for the straight cylinder at broadside incidence, the trend increases with frequency (actually $\mathrm{ka}$ ) because of the fact that this cylinder is curved only in one dimension. This effect is related to the fact that the size of the cylinder remains much smaller than the first Fresnel zone in these (finite cylinder) calculations (Stanton, 1988). Once the frequencies are high enough or the range to the target small enough, then many Fresnel zones occupy the cylinder and the cylinder appears acoustically like an infinitely long cylinder (DiPerna and Stanton, 1991). The trend in this latter case levels off as with the other bodies (not shown). For end-on incidence, the scattering is due to the rounded ends of the cylinders and the trend is constant, but at a lower level since the cross section of the ends is relatively small.

Once the scattering is averaged over angle of orientation, some of the differences between the scattering by the sphere and cylinders tend to diminish. This is due to the directional nature of the scattering by the cylinders: for a single orientation, a substantial portion of the scattered signals from a cylinder may or may not be in the direction back toward the receiver. However, once averaged over all angles of orientation, the orientations where substantial backscattered energy occurs become part of the average, hence reducing the differences between the average scattering by the different bodies. For example, at broadside incidence, the scattering by a straight cylinder may be stronger than that of 
the bent cylinder at broadside incidence. For orientations away from broadside incidence, the scattered signal from the straight cylinder will diminish much more rapidly than that from the bent cylinder because the width of the main lobe of the scatter pattern of the straight cylinder is narrower than that of the bent cylinder of the same length. Hence, the straight cylinder has a stronger but narrower main lobe compared with that of the bent cylinder. The average over all angles gives nearly the same value for each type of cylinder, indicating that the change in shape of the main lobes offsets the differences in overall levels within the main lobes. This phenomenon, in essence, is related to the principle of conservation of energy (Stanton et al., 1993b). [Of course, averaging over narrower distributions can sometimes result in differences as well, as illustrated, for example, in Figs. 2 and 3 of this paper, Stanton et al. (1993b), and Demer and Martin (1995).]

Another effect in the averaging over size and angle of orientation involves the smoothing out of the oscillatory pattern of target strength versus frequency. For the sphere, this effect of smoothing out is due solely to the fact that the positions of the nulls and peaks of the pattern are related to the radius of the body. The pattern is slightly different from realization to realization in the averaging over sizes, and hence the null value of one realization will be averaged with higher values from other realizations, which will tend to fill in the nulls. The nulls become increasingly affected for high frequencies as a given change in size will be larger with respect to wavelength at the higher frequencies.

For the cylinders, the pattern of target strength versus frequency is dependent upon both size (as with the sphere) as well as orientation. Consequently, averages over both size and orientation cause reduced structure in the pattern. However, because of the fact that the backscattering values at broadside incidence are much greater than those at end-on, the pattern near broadside incidence will tend to dominate the scattering. Still, the average over sizes affects the pattern as much as with the sphere.

While the averaging reduces differences between the scattering by the bodies of various shapes, the scattering still depends upon the particular distribution of orientation of the bodies. For averages over the distribution of angles of orientation $\mathrm{N}\left(20^{\circ}, 90^{\circ}\right)$ for the cylinders, the scattering levels were similar for the cylinders and spheres in the geometric region (Fig. 3). For other distributions, the averages were sometimes quite different, especially when the mean angle of orientation of the cylinders was well away from broadside incidence (Fig. 2). For distributions of $\mathrm{N}\left(20^{\circ}, 20^{\circ}\right), \mathrm{N}\left(20^{\circ}, 60^{\circ}\right)$, and uniform $(0-2 \pi)$, the scattering is within several $\mathrm{dB}$ of that of the sphere at high frequencies (Fig. 2).

\section{FIELD IMPLICATIONS}

The results show that for high enough acoustic frequencies and certain distributions of angle of orientation, interpretation of surveys of elongated animals is relatively insensitive to the choice of model shape (i.e., sphere versus cylinder). However, for lower frequencies or other behavioral conditions, the animal shape and orientation distribution need to be taken into account. For example, interpreta-
TABLE II. Number of objects per cubic meter it would require to produce a volume scattering strength of $-70 \mathrm{~dB}$. The volume of each object is fixed at $0.30 \mathrm{~cm}^{3}$. The angular distributions of the bent cylinders are varied as indicated at the top of the columns. The models, body dimensions, average over size, and values of $g, h$, and $\rho_{c} / L$ (cylinders only) are the same as in Fig. 2. The frequencies chosen correspond to those of commercially available echosounders.

\begin{tabular}{rcccccc}
\hline \hline & \multicolumn{5}{c}{ Cylinder } \\
$\begin{array}{rlc}\text { Freq. } \\
(\mathrm{kHz})\end{array}$ & Sphere & $\begin{array}{c}\mathrm{N} \\
\left(20^{\circ}, 20^{\circ}\right)\end{array}$ & $\begin{array}{c}\mathrm{N} \\
\left(30^{\circ}, 20^{\circ}\right)\end{array}$ & $\begin{array}{c}\mathrm{N} \\
\left(40^{\circ}, 20^{\circ}\right)\end{array}$ & $\begin{array}{c}\mathrm{N} \\
\left(50^{\circ}, 20^{\circ}\right)\end{array}$ & $\begin{array}{c}\text { Uniform } \\
{\left[0,360^{\circ}\right]}\end{array}$ \\
\hline 38 & 27 & 50 & 76 & 140 & 280 & 160 \\
120 & 31 & 3.3 & 5.6 & 14 & 55 & 11 \\
200 & 12 & 4.9 & 8.2 & 20 & 60 & 16 \\
420 & 11 & 5.9 & 9.9 & 24 & 74 & 19 \\
\hline \hline
\end{tabular}

tion of the echoes from a $38-$ or $120-\mathrm{kHz}$ single frequency system could be affected by about $5 \mathrm{~dB}$ when surveying 34-mm-long euphausiids with a $\mathrm{N}\left(20^{\circ}, 90^{\circ}\right)$ distribution while the work at 200 or $420 \mathrm{kHz}$ would be relatively unaffected for that length of animals (Fig. 3). For a distribution of $\mathrm{N}\left(45^{\circ}, 20^{\circ}\right)$ the interpretation for $34-\mathrm{mm}$-long euphausiids is affected by about $6-7 \mathrm{~dB}$ for most frequencies at or above $38 \mathrm{kHz}$, except for frequencies near $120 \mathrm{kHz}$ where the differences are much less (Fig. 2). These types of (decibel) errors can translate into errors in estimates of the numbers of animals causing the scattering (Table II).

Success of a two- or multi-frequency system is also affected by the shape and behavioral conditions. Each approach not only depends upon the value of the scattering levels, but the "transition point"' between the Rayleigh and geometric scattering region (Greenlaw, 1979; Holliday and Pieper, 1995). A crucial phenomenon illustrated in these calculations is the fact that the transition point for the sphere is different from that of a cylinder (Fig. 3). For example, for a 29 -mm-long cylinder, the point is at about $150 \mathrm{kHz}$. For a sphere with the same volume as that of a 29-mm-long cylinder, the point is at about $75 \mathrm{kHz}$. Hence the transition points are different by a factor of 2 . This difference would have a profound effect on an analysis or algorithm that relies on knowing where the transition point is.

\section{SUMMARY AND CONCLUSIONS}

Comparisons between the scattering by weakly scattering spheres and cylinders of the same volume have shown similar or identical levels under certain limiting conditions and dramatic differences under other conditions. The levels are identical in the limit of very low frequencies when the product of the wave number and all outer dimensions of the body is much less than unity. However, that region is not particularly useful because the echo levels tend to be negligibly small in practical survey systems. For moderately low frequencies $(k a \ll 1, k L \geqslant 1)$ and higher, there are distinct differences between the scattering levels of the different bodies due to the elongated nature of the cylinders and orientation effects. For certain orientation distributions, the averaged scattering levels of all bodies are very close to each other in the geometric scattering region. Other distributions produce substantially different average levels between the sphere and 
cylinders. Regardless of orientation distribution, the turning point between Rayleigh and geometric scattering occurs at different frequencies for the bodies.

These results show that for surveys of elongated animals in the ocean, the averaged echo energies (say, from an echo integrator) could be relatively insensitive to shape at high enough acoustic frequencies (for example, greater than 300 $\mathrm{kHz}$ for a 34-mm-long euphausiid) and for certain distributions of angles of orientation. However, for surveys involving other distributions of angles of orientations at high frequencies or lower frequencies (the "moderately low frequency range", which would be, for example, 20-200 $\mathrm{kHz}$ for 34-mm-long euphausiids), the results become strongly dependent upon shape and the modeling must distinguish between spherical and cylindrical animals.

Also, while the focus of this work involved shape dependencies of acoustic scattering for bodies with the same material properties, variations in material properties also significantly affect the scattering levels as briefly discussed herein [see, for example, Stanton et al. (1994) for data and modeling of animals with various material properties as well as references in that paper to other works on the subject].

In conclusion, as observed in this study and the many studies referenced herein, the scattering of sound is a complex function of size, shape, orientation, and material properties of the body as well as acoustic wavelength. Ideally, one should take each factor into account in scattering predictions as accurately as possible. However, some of these factors may be more important than others, depending upon conditions such as which scattering region the object is in (Rayleigh/geometric) or whether or not the echoes are being averaged over a particular distribution of angles of orientation. Analysis of the scattering therefore requires determination of the conditions and which factors (such as shape and orientation distribution) need to be taken into account in the modeling.

\section{ACKNOWLEDGMENTS}

The authors are grateful to Shirley (Bowman) Barkley for preparing the manuscript to this paper. This work was supported by the U.S. Office of Naval Research Grant No. N00014-95-1-0287 and the National Science Foundation Grant No. OCE-9201264. This is WHOI contribution No. 9467.

Anderson, V. C. (1950). "Sound scattering from a fluid sphere," J. Acoust. Soc. Am. 22, 426-431.

Born, M., and Wolf, E. (1991). Principles of Optics (Pergamon, New York). Chu, D., Foote, K. G., and Stanton, T. K. (1993). "Further analysis of target strength measurements of Antarctic krill at $38 \mathrm{kHz}$ and $120 \mathrm{kHz}$ : Comparison with deformed cylinder model and inference of orientation distribution," J. Acoust. Soc. Am. 93, 2985-2988.

Clay, C. S., and Medwin, H. (1977). Acoustical Oceanography: Principles and Applications (Wiley-Interscience, New York).
Demer, D. A., and Martin, L. V. (1995). "Zooplankton target strength: Volumetric or areal dependence?'” J. Acoust. Soc. Am. 98, 1111-1118.

DiPerna, D. T., and Stanton, T. K. (1991). "Fresnel zone effects in the scattering of sound by cylinders of various lengths," J. Acoust. Soc. Am. 90, 3348-3355.

Endo, Y. (1993). “'Orientation of Antarctic Krill in an aquarium,' Nippon Suisan Gakkaishi 59, 465-468.

Foote, K. G. (1990). "Speed of sound in Euphausia superba,' J. Acoust. Soc. Am. 87, 1405-1408.

Foote, K. G., Everson, I., Watkins, J. L., and Bone, D. G. (1990). "Target strengths of Antarctic krill (Euphausia superba) at 38 and $120 \mathrm{kHz}$, , J. Acoust. Soc. Am. 87, 16-24.

Gaunaurd, G. C. (1985). "Sonar cross sections of bodies partially insonified by finite sound beams," IEEE J. Ocean Eng. OE-10, 213-230.

Greenlaw, C. F. (1977). "Backscattering spectra of preserved zooplankton,', J. Acoust. Soc. Am. 62, 44-52.

Greenlaw, C. F. (1979). "Acoustical estimation of zooplankton populations," Limnol. Oceanogr. 24, 226-242.

Holliday, D. V., and Pieper, R. E. (1995). "Bioacoustical oceanography at high frequencies,' ICES J. Mar. Sci. 52, 279-296.

Holliday, D. V., Pieper, R. E., and Kleppel, G. S. (1989). "Determination of zooplankton size and distribution with multi-frequency acoustic technology,' J. Conseil Int. L'Explor. Mer. 46, 52-61.

Johnson, R. K. (1977). "Sound scattering from a fluid sphere revisited," J. Acoust. Soc. Am. 61, 375-377; " Erratum: 'Sound scattering from a fluid sphere revisited',"' J. Acoust. Soc. Am. 63, 626 (1978).

Kils, U. (1981). "The swimming behavior, swimming performance and energy balance of Antarctic krill, Euphausia superba," BIOMASS Science Series Vol. 3.

Marston, P. L. (1992). "Geometrical and catastrophe optics methods in scattering," in Physical Acoustics, edited by A. D. Pierce and R. N. Thurston (Academic, New York), Vol. 21.

Miyashita, K., Aoki, I., and Inagaki, T. (1996). "Swimming behavior and target strength of isada krill (Euphausia pacifica),' ICES J. Mar. Sci. 53, 303-308.

Morse, P. M., and Ingard, K. U. (1968). Theoretical Acoustics (Princeton U. P., Princeton, NJ), Chap. 8.

Ogilvy, J. A. (1991). Theory of Wave Scattering from Random Rough Surfaces (Adam Hilger, New York).

Partridge, C., and Smith, E. R. (1995). "Acoustic scattering from bodies: Range of validity of the deformed cylinder method,' J. Acoust. Soc. Am. 97, 784-795.

Stanton, T. K. (1988). "Sound scattering by cylinders of finite length. I. Fluid cylinder,' J. Acoust. Soc. Am. 83, 55-63.

Stanton, T. K. (1989). "Sound scattering by cylinders of finite length. III. Deformed cylinders," J. Acoust. Soc. Am. 86, 691-705.

Stanton, T. K. (1992). "Sound scattering by rough elongated elastic objects: I. Means of scattered field,' J. Acoust. Soc. Am. 92, 1641-1664.

Stanton, T. K., Chu, D., and Wiebe, P. H. (1998). "Sound scattering by several zooplankton groups. II. Scattering models,' J. Acoust. Soc. Am. 103, 236-253.

Stanton, T. K., Clay, C. S., and Chu, D. (1993a). "Ray representation of sound scattering by weakly scattering deformed fluid cylinders: Simple physics and application to zooplankton," J. Acoust. Soc. Am. 94, 34543462 .

Stanton, T. K., Chu, D., Wiebe, P. H., and Clay, C. S. (1993b). “Average echoes from randomly oriented random-length finite cylinders: Zooplankton models," J. Acoust. Soc. Am. 94, 3463-3472.

Stanton, T. K., Nash, R. D. M., Eastwood, R. L., and Nero, R. W. (1987). "A field examination of acoustical scattering from marine organisms at 70 kHz,' IEEE J. Ocean. Eng. OE-12(2), 339-348.

Stanton, T. K., Wiebe, P. H., Chu, D., Benfield, M. C., Scanlon, L., Martin, L., and Eastwood, R. L. (1994). "On acoustic estimates of zooplankton biomass," ICES J. Mar. Sci. 51, 505-512.

Urick, R. J. (1983). Principles of Underwater Sound (McGraw-Hill, New York). 